\title{
An update on adjuvant systemic therapies in melanoma
}

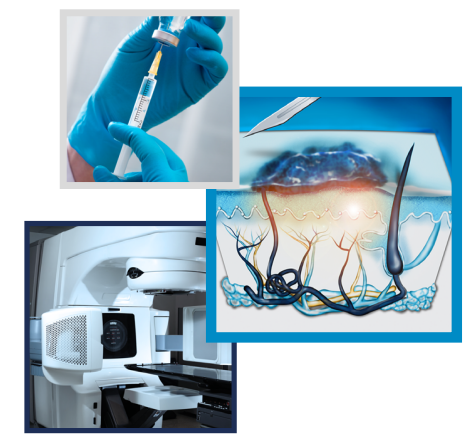

\author{
Evangeline Samuel*,1(D), Maggie Moore², Mark Voskoboynik² (D), Mark Shackleton² \& \\ Andrew Haydon ${ }^{2}$ \\ ${ }^{1}$ Department of Medical Oncology, Monash Health, Clayton, Melbourne 3168, Australia \\ ${ }^{2}$ Department of Medical Oncology, The Alfred Hospital, Melbourne 3004, Australia \\ *Author for correspondence: evangeline_nj@yahoo.co.in
}

There is a global increase in the incidence of melanoma, with approximately 300,000 new cases in 2018 worldwide, according to statistics from the International Agency for Research on Cancer. With this rising incidence, it is important to optimize treatment strategies in all stages of the disease to provide better patient outcomes. The role of adjuvant therapy in patients with resected stage 3 melanoma is a rapidly evolving field. Interferon was the first agent shown to have any utility in this space, however, recent advances in both targeted therapies and immunotherapies have led to a number of practice changing adjuvant trials in resected stage 3 disease.

First draft submitted: 3 March 2019; Accepted for publication: 19 June 2019; Published online: 13 November 2019

Keywords: adjuvant treatment $\bullet$ AJCC • immunotherapy • interferon • ipilimumab • stage 3 melanoma $\bullet$ targeted therapy

Although the survival of early-stage melanoma is very good, the prognosis of stage 3 disease is considerably poorer. Five-year overall survival (OS) for patients with lymph node (LN) metastases is 63\%, and with macroscopic nodal involvement, this drops to $43 \%$ [1]. The last decade has witnessed a paradigm shift in the treatment landscape of metastatic melanoma, with the advent of targeted and immunotherapies. Therapeutic strategies that are effective in the metastatic setting are often then studied in the adjuvant context. By eliminating the micrometastatic disease that remains after surgery, adjuvant systemic therapy aims to reduce disease recurrence and ultimately improve rates of cure following surgical resection of locoregional disease. This strategy has been widely and successfully used by oncologists treating many different tumor types over the last five decades.

For many years, systemic therapies for advanced melanoma had been ineffective, with no treatment demonstrating a benefit in survival in stage 4 patients prior to 2010. As a result, standard management of stage 3 melanoma was surgery followed by observation, with no internationally accepted adjuvant treatment. This approach, however, is rapidly changing, with practice-changing results from adjuvant trials. This review will outline the major developments in the adjuvant systemic treatment of melanoma.

\section{Interferon}

IFN- $\alpha-2 b$ exhibits modest antitumor activity with response rates of $12-16 \%$ in metastatic melanoma, with the exact mechanism of action not completely understood [2]. Multiple studies of IFN- $\alpha-2 b$, including various dosing schedules, have been performed in Europe and the USA, with most failing to show an OS benefit.

The Eastern Cooperative Oncology Group (ECOG), in a randomized controlled trial (ECOG 1684), showed a significant benefit in relapse-free survival (RFS), with an absolute improvement of $11 \%(\mathrm{p}=0.0023$, one-sided) and a $9 \%$ absolute improvement in OS ( $\mathrm{p}=0.0237$, one-sided) at 5 years, respectively, with IFN- $\alpha-2 \mathrm{~b}$ compared with observation alone. There were greater benefits seen in the node-positive group [3]. However, with longer follow-up at 12.6 years no survival benefit was seen [4].

While the US investigators were using high-dose IFN for 12 months, investigators in Europe were testing lowto medium-dose IFN for up to 5 years [5]. The EORTC combined results of two studies, EORTC 18952 and 18991 into a small meta-analysis, using individual patient data. This showed that the hazard ratio (HR) for OS of 
adjuvant IFN therapy was 0.77 (99\% CI: 0.63-0.93); in patients with ulcerated primaries, however, there was no impact of adjuvant IFN in the 2118 patients without ulceration (HR: 0.98; 99\% CI: 0.87-1.17) [6]. The treatment interaction between ulceration and PEG-IFN was also investigated in the Sunbelt trial, which enrolled patients with microscopic nodal involvement only, finding no effect of adjuvant IFN in non-ulcerated patients but significant benefit in patients with ulcerated primary tumors [7].

In a meta-analysis by Mocellin et al. of 17 randomized controlled trials (RCTs), benefits of adjuvant IFN were observed in improving the disease-free survival (HR: 0.83; 95\% CI: $0.78-0.87, \mathrm{p}<0.00001$ ) and to a lesser extent the OS (HR: $0.91 ; 95 \%$ CI: $0.85-0.97 ; \mathrm{p}=0.003$ ), with no significant interstudy heterogeneity [8]. This modest survival benefit ( $3-4 \%$ improvement in absolute 5 -year OS) balanced against toxicity and cost has limited the uptake of IFN as an adjuvant treatment for melanoma.

\section{Immunotherapy}

Ipilimumab

Discovery of regulatory pathways that limit immune responses to cancer has led to landmark evolution in the development of anticancer therapies. CTLA- 4 has a crucial role in immune checkpoint regulation, by downregulating T-cell activation [9]. Ipilimumab, by inhibiting the CTLA- 4 molecule, enhances antitumor immune responses. This drug is proven to have a role in metastatic melanoma in Phase III studies [10,11]. One trial suggested increased efficacy against metastatic disease with a dose of $10 \mathrm{mg} / \mathrm{kg}$ compared with $3 \mathrm{mg} / \mathrm{kg}$, albeit at the expense of higher toxicity [12].

These findings led to the EORTC 18071 study, a Phase III trial of ipilimumab versus placebo in patients with completely resected stage 3A (if LN metastasis $>1 \mathrm{~mm}$ ), 3B or 3C melanoma [13]. Patients with in-transit metastasis were excluded. Disease staging was according to the American Joint Committee on Cancer (AJCC) 7th edition [14].

In this randomized, double-blind, multicenter trial, eligible patients were randomly assigned to receive an intravenous infusion of ipilimumab at a dose of $10 \mathrm{mg} / \mathrm{kg}$ or placebo in a 1:1 ratio. They received this treatment every 3 weeks for four doses, then 3 monthly for up to 3 years or until disease recurrence or unacceptable toxicities. Patients were required to have undergone a complete regional lymphadenectomy within 12 weeks prior to randomization. The primary end point of this trial was RFS and secondary end points of interest included OS and distant metastasis-free survival.

At 5 years, the trial showed a $10 \%$ absolute improvement in OS (65.4 vs 54.4\%), (HR for death: 0.72; $95.1 \%$ CI: $0.58-0.88 ; \mathrm{p}=0.001$ ), RFS (40.8 vs $30.3 \%$ ) (HR for recurrence or death: 0.76 ; $95 \%$ CI: $0.64-0.89$; $\mathrm{p}$ $<0.001$ ) and distant metastasis-free survival (48.3 vs 38.9\%) (HR for death or distant metastasis: 0.76 ; $95.8 \%$ CI: $0.64-0.92 ; \mathrm{p}=0.002)$. Subgroup analysis failed to demonstrate a significant interaction between ulceration, number of LNs involved or type of LN involvement (microscopic vs macroscopic) [15].

Toxicity has limited widespread adoption of this regimen by oncologists. Only $13.4 \%$ of patients completed the full planned course of treatment, and nearly $40 \%$ of patients discontinued treatment after the first four doses due to treatment-related side effects. The rates of grade 3 or 4 adverse effects (AEs) were $54.1 \%$ in the ipilimumab arm, with five $(1.1 \%)$ treatment-related deaths. The high rates of death and adverse events have raised caution in employing this treatment routinely in the adjuvant setting. Despite the high rates of toxicity, there were surprisingly no quality of life (QOL) differences between the two treatment arms as per the EORTC QLQ-C30 GH/QoL score. However, diarrhea, fatigue and insomnia were associated with ipilimumab at week 10.

PD-1

The programmed cell death 1 (PD-1) receptor is able to inactivate activated $\mathrm{T}$ cells reaching tumors by engaging with its ligand PD-L1, which is expressed in peripheral tissues and cancer cells [16]. Two monoclonal antibodies targeting this checkpoint inhibitory pathway, pembrolizumab, and nivolumab, showed effective durable responses in the treatment of metastatic melanoma and replaced ipilimumab monotherapy as standard first-line treatment of stage 4 melanoma [17-21]. Adjuvant anti-PD-1 therapy has been tested in two large Phase III studies - Checkmate 238 and Keynote 054.

The Checkmate 238 study segued from the EORTC 18071 study comparing nivolumab to the control arm of high-dose ipilimumab. In this randomized, Phase 3, double-blind trial, 906 patients who had undergone complete resection of stage 3B, 3C or 4 melanoma were randomized in a 1:1 ratio to receive either intravenous nivolumab or ipilimumab. Nivolumab was given 2 weekly at a dose of $3 \mathrm{mg} / \mathrm{kg}$ and ipilimumab every 3 weeks, at a dose of 
$10 \mathrm{mg} / \mathrm{kg}$. All patients received a total of four doses, followed by doses every 12 weeks. Treatment was continued for up to 1 year, or until the development of unacceptable side effects or relapse.

The study met its primary end point in demonstrating a significant improvement from nivolumab in RFS (HR: 0.65 ; $97.56 \%$ CI: $0.51-0.83$; p < 0.001), equating to a $10 \%$ absolute improvement at 12 months. Grade $3-4$ treatment-related AEs were much higher in the ipilimumab arm 45.9\%, compared with the nivolumab arm $14.4 \%$, with higher AEs leading to treatment discontinuation in the ipilimumab arm (42 vs 8\%) [22]. Nivolumab was not associated with any treatment-related toxic deaths.

The benefit was seen in all subgroups including different disease stages, microscopic versus macroscopic nodal disease, ulceration status of the primary tumor, BRAF status and PD-L1 status. The benefit of nivolumab was seen in stage 3 disease (HR: $0.65,0.52-0.83$ ), with a strong trend toward improvement in resected stage 4 patients (HR: $0.70,0.45-1.10)$. Mature results are awaited to ascertain if this early promising result will translate into an OS benefit.

The Keynote 054 trial (EORTC 1325) was a randomized, double-blind, Phase III trial, in an identical patient population to the EORTC 18071 trial. It compared pembrolizumab (200 mg every 3 weeks) with placebo as adjuvant therapy in resected, high-risk stage 3 melanoma. Higher 12-month RFS was observed in the pembrolizumab group 75.4\% (95\% CI: 71.3-78.9) compared with placebo 61.0\% (95\% CI: 56.5-65.1), with a HR for RFS of 0.57 (98.4\% CI: 0.43-0.74; p < 0.001; Figure 1) [23]. Further follow-up is required to assess OS. Grade 3-5 treatmentrelated AEs were reported in $14.7 \%$ of patients in the pembrolizumab group and in $3.4 \%$ in the placebo group. The rates of treatment discontinuation due to an adverse event was $13.0 \%$ in the pembrolizumab group compared with $1.8 \%$ in the placebo group. One treatment-related death due to myositis was observed in the pembrolizumab group. Health-related quality of life (HRQOL) analysis showed no clinically meaningful differences between the two arms, despite the higher incidence of AEs in the treatment arm [24].

Importantly, the Keynote 054 trial allowed for crossover of patient's in the placebo arm to the pembrolizumab arm, when they relapsed. This design will address the important question of whether adjuvant immunotherapy with pembrolizumab is superior to treatment at recurrence.

These two trials have played a key role in changing practice in the adjuvant setting, making PD-1 inhibitor treatment an option after resection of stage 3 melanoma. Although mature survival data are needed, PD-1 inhibition

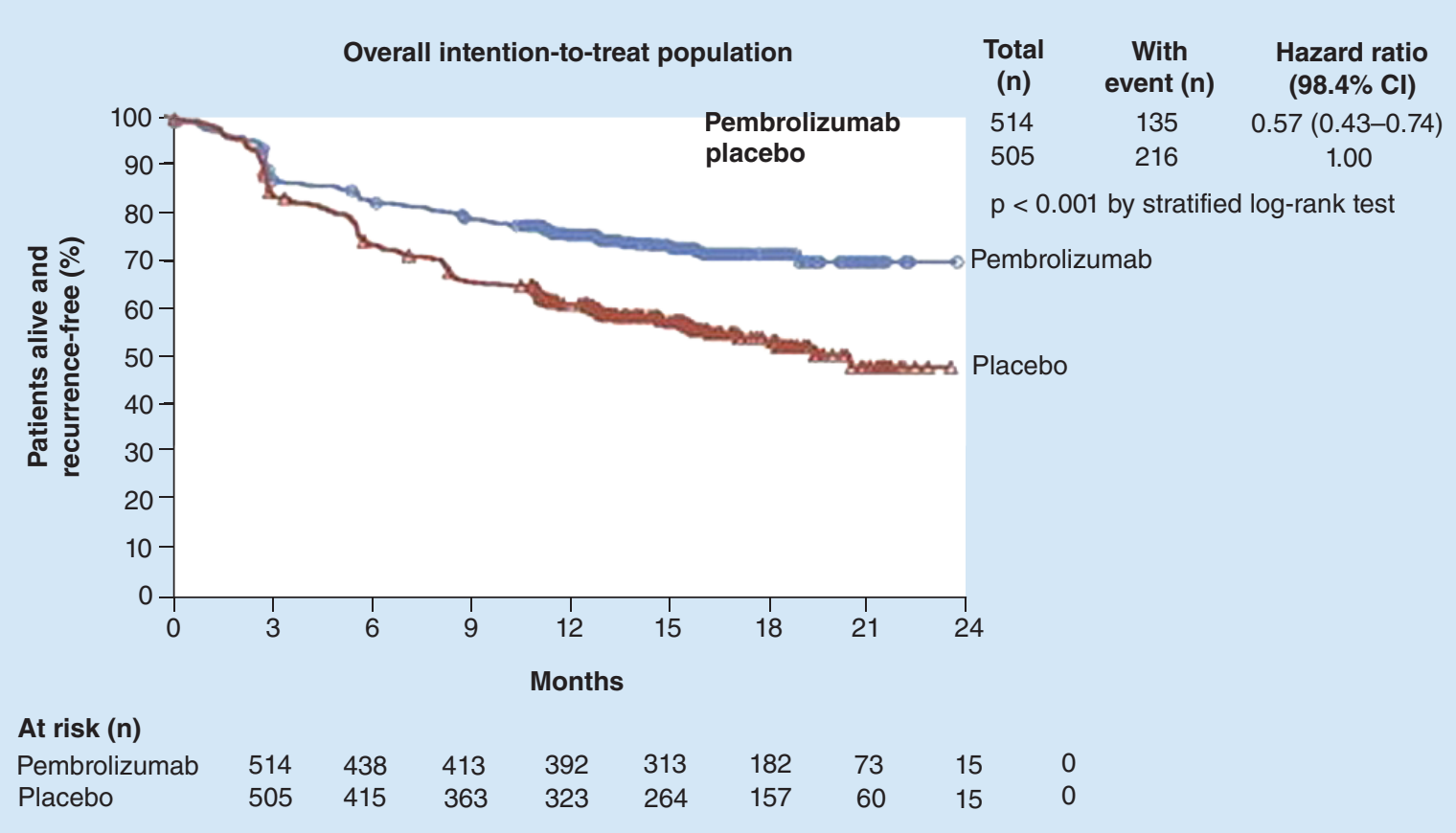

Figure 1. Kaplan-Meier estimate of recurrence-free survival in the overall intention-to-treat population in those who received pembrolizumab compared with placebo. 
is now being offered to patients in the adjuvant setting based on the consistent, substantial and clinically meaningful improvements in RFS.

\section{Adjuvant targeted therapies}

The discovery of oncogenic molecular pathways in melanoma has led to the identification of targetable mutations. Mutations of the $B R A F$ gene is one such target and is found in approximately $40 \%$ of melanomas. This results in spontaneous activation of the MAPK pathway that encompasses the $R A S / R A F / M E K / E R K$ genes, thus making this pathway a therapeutic target in melanoma [25].

Accordingly, the development of BRAF inhibitors and MEK inhibitors has allowed for combined inhibition of the MAPK pathway and has led to practice changing improvements in outcomes for patients with metastatic BRAF mutant melanoma. The BRIM-3 and BREAK-3 trials showed improved OS with single-agent BRAF inhibitors (vemurafenib and dabrafenib respectively) compared with DTIC [26,27]. Shortly after, COMBI-V, COMBI-D, COLUMBUS and CO-BRIM all showed the superiority of combination BRAF + MEK inhibitors over singleagent BRAF inhibitors [28-31]. These results paved the way for adjuvant clinical trials of MAPK inhibition in high-risk melanoma

COMBI-AD is a Phase III, double-blind, placebo-controlled trial that enrolled patients with completely resected, stage 3 melanoma harboring BRAF V600E or V600K mutations. A total of 870 patients were randomized to receive oral dabrafenib at a dose of 150-mg twice daily plus trametinib at a dose of 2-mg once daily or placebo. Treatment was continued up to 1 year. Patients with stage 3A ( $>1 \mathrm{~mm} \mathrm{LN}$ metastasis), 3B or 3C disease were eligible and treatment commenced within 12 weeks of surgery.

COMBI-AD showed a 3-year absolute improvement in RFS of 19\% (HR for relapse or death: $0.47 ; 95 \%$ CI: 0.39-0.58; $\mathrm{p}<0.001$; Figure 2) and an absolute OS benefit of 7\% (HR for death: 0.57; 95\% CI: 0.42-0.79; $\mathrm{p}=0.0006$ ), respectively. However, the level of improvement in OS did not cross the prespecified threshold of $\mathrm{p}$ $=0.000019$ to make OS results statistically significant, at the first interim analysis. The rates of grade 3 or 4 AEs

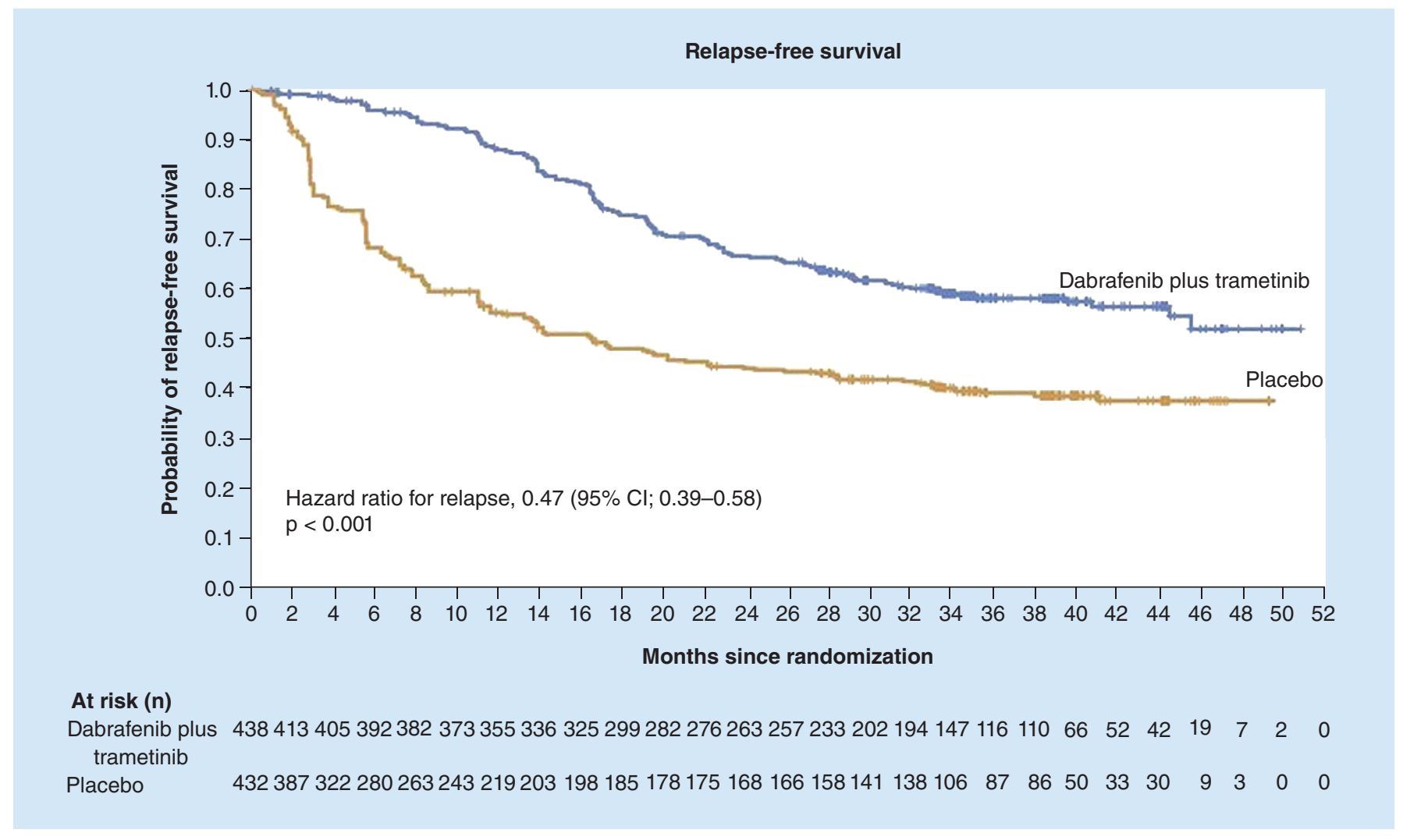

Figure 2. Kaplan-Meier estimates of relapse-free survival among the patients who received combination therapy with dabrafenib plus trametinib and those who received placebo in the intention-to-treat analysis. 
were $41 \%$ in the combination arm and $14 \%$ in the placebo group, with pyrexia, fatigue and nausea being the most common toxicities associated with the combination [32]. Twenty-six percent of patients stopped treatment early due to an AE. However, the formal QOL evaluation showed no clinically significant differences between the two arms, including in those who experienced a higher frequency of AEs in the treatment arm [33].

Extended follow-up to 40 months has demonstrated a sustained and significant 16\% absolute difference in RFS between the two arms (HR: 0.49; 95\% CI: 0.40-0.59). This benefit is similar in all subgroups, for example, disease substage, metastatic load and tumor ulceration status [34].

The alternative first-generation BRAF inhibitor, vemurafenib, was evaluated in the BRIM8 study, another Phase III, double-blind trial that randomized 498 patients between oral vemurafenib ( $960 \mathrm{mg}$ twice daily) or placebo in completely resected stage 2C-3A-3B (cohort 1) or stage 3C (cohort 2) BRAF V600 mutation-positive melanoma. Again, treatment was given for up to 12 months. The primary end point was disease-free survival in the intentionto-treat population, evaluated separately in each cohort. Hierarchical analysis of cohort 2 before cohort 1 was prespecified in the trial. The trial results were deemed nonsignificant because there was no meaningful DFS benefit in cohort 2 (3C disease) (23.1 months [95\% CI: 18.6-26.5] in the vemurafenib group vs 15.4 months [11.1-35.9] in the placebo group [HR: 0.80; 95\% CI: $0.54-1.18$; $\log$-rank $\mathrm{p}=0.26$ ]) at median follow-up of 33.5 months. In cohort 1 (patients with stage 2C-3A-3B disease), median disease-free survival was not reached in the vemurafenib group versus 36.9 months in the placebo group (HR: 0.54; 95\% CI: 0.37-0.78; log-rank p = 0.0010). Grade 3-4 AEs occurred in $57 \%$ of patients in the vemurafenib group and $15 \%$ of patients in the placebo group, with the most common being keratoacanthomas, arthralgia and squamous cell carcinoma [35].

\section{Additional points for consideration}

Implications of the AJCC 8th edition staging system

It is important to note that patients in the adjuvant immunotherapy and targeted therapy studies were all staged according to the 7 th edition of the AJCC staging system, as the 8th edition was released only in early 2018 . Unlike the 7 th edition, the 8 th edition takes into account $\mathrm{T}$ stage in addition to the $\mathrm{N}$ stage to determine the substage of stage 3 melanoma and divides patients into four subgroups (stage $3 \mathrm{~A}, 3 \mathrm{~B}, 3 \mathrm{C}$ as well as the new stage $3 \mathrm{D}$ category) [36].

A post hoc analysis of the COMBI-AD trial was conducted to analyze the benefits of this treatment based on classification of baseline disease stages according to the new AJCC 8th edition. This showed that the RFS benefits were consistent across all subgroups of the AJCC 8th edition, when compared with placebo (stage 3A - HR: 0.63; 95\% CI: 0.26-1.56; stage 3B - HR: 0.48; 95\% CI: 0.34-0.67; stage 3C - HR: 0.50; 95\% CI: 0.38-0.64; stage 3D - HR: 0.34; 95\% CI: 0.14-0.79) [37]. A similar reanalysis was performed for the Keynote 054 study. The 1-year RFS rate for pembrolizumab versus placebo and the HRs (99\% CI) within each AJCC-8 subgroup were stage $3 \mathrm{~A}$ (92.7 vs $92.5 \%$; 0.76 [0.11-5.43]), 3B (79.0 vs $65.5 \%$; 0.59 [0.35-0.99]), 3C (73.6 vs $53.9 \%$; 0.48 $[0.33-0.70])$ and $3 \mathrm{D}(50.0$ vs $33.3 \% ; 0.69$ [0.24-2.00]) [38]. Only $10 \%$ of patients enrolled into COMBI-AD and $8 \%$ of patients in Keynote 054 had stage 3 A disease according to the new staging system.

These data suggest that caution should be taken when recommending adjuvant therapy to patients with stage 3A melanoma (based on the AJCC 8th edition). Although the test for an interaction between improved RFS and substage according to the AJCC 8th edition was not significant in either study, the good prognosis and very small benefit seen in patients with stage $3 \mathrm{~A}$ melanoma suggest at best a modest therapeutic benefit in this subgroup. This consideration is especially relevant when discussing adjuvant immunotherapy that may cause long-term toxicities.

\section{The role of completion LN dissection}

The multicenter selective lymphadenectomy (MSLT) - II [39] and De COG [40] trials have led to changes in current practice, with respect to LN dissection. There were no improvements seen with respect to melanoma-specific OS in patients who underwent completion LN dissection compared with observation alone complemented by routine ultrasound surveillance, in these studies. The benefit was limited to locoregional disease control only. These data are notable, as the adjuvant trials discussed above, which were designed and carried out prior to these trials and mandated a formal nodal clearance prior to adjuvant therapy. Although completion LN dissection is no longer considered necessary following the discovery of occult disease in a sentinel LN, there is no reason to expect the benefits seen with new adjuvant therapies would not apply to patients who do not undergo a completion LN dissection. Adjuvant therapy has been shown to reduce both local relapses and distant recurrences, however, all of the studies involving immunotherapy or targeted therapy mandated completion LN dissections in all patients 
with stage 3 disease. As such evidence is lacking for patients who do not undergo a completion LN dissection and requires ongoing investigation.

\section{Discussion}

Major advances in the treatment of metastatic melanoma over recent years have led to a number of practice changing Phase III trials in the adjuvant treatment for melanoma. Keynote 054, Checkmate 238 and COMBI-AD have demonstrated unprecedented improvements in RFS for stage 3 melanoma that are achieved with substantially less toxicity than high-dose IFN or ipilimumab.

The EORTC 18071, Keynote 054 and COMBI-AD trials all enrolled patients who had resected regional LNpositive (stage III) melanoma with a high risk of recurrence. This included stages $3 \mathrm{~A}$ (if LN metastasis $>1 \mathrm{~mm}$ ), $3 \mathrm{~B}$ or $3 \mathrm{C}$ disease with no in-transit metastasis. However, these trials do not address the effect of adjuvant treatment in stage $3 \mathrm{~A}$ patients with $\mathrm{LN}$ metastasis $<1 \mathrm{~mm}$. As such, it is necessary to extrapolate the data to this population. This is not unreasonable given the consistency of the HRs seen across the various stage 3 subclassification, although the absolute magnitude of benefit of adjuvant therapy is smaller with better prognosis disease.

One important question remaining is whether with longer follow-up the effect of adjuvant PD-1 immunotherapy on OS will follow the improved RFS already evident. Given the ability of immunotherapy to cause long-term disease control (and possibly cure in some cases) in a substantial proportion of stage 4 melanoma patients, it is possible that the patients who benefit from adjuvant PD-1 therapy may derive similar long-term benefit with treatment at relapse. The design of the Keynote 054 study (with crossover to pembrolizumab provided for patients relapsing on placebo) should answer this very important question. The fact that adjuvant ipilimumab in the EORTC 18071 resulted in an OS improvement is proof of principle that adjuvant immunotherapy can improve survival, however, this study was carried out in an era prior to the widespread availability of anti-PD-1 for metastatic melanoma [41].

Another unanswered question is whether adjuvant anti-PD-1 immunotherapy or adjuvant dabrafenib and trametinib are preferred agents for stage 3 BRAF mutant melanoma. Both approaches show similar improvements in RFS with an absolute reduction in relapse rates of $15-20 \%$. Relapses on treatment (in the 1st year following randomization) were more frequent with immunotherapy; however, more relapses occur in the 2nd year when targeted therapy is used. To date, only COMBI-AD has reported OS data, with a 7\% advantage after 2.8 years follow-up and the RFS benefit is maintained at a longer follow-up to 4 years. OS results from Checkmate 238 and Keynote 054 are still pending. So at present, there is no clear evidence to guide management in this subgroup. Most clinicians feel that anti-PD-1 immunotherapy will be a superior treatment in this group of patients, mainly driven by their experience in treating stage 4 disease. More mature data are needed to address this issue. The other important factor to weigh up relates to the toxicities of these two treatment options. Dabrafenib and trametinib resulted in higher rates of grade 3-4 adverse events than nivolumab or pembrolizumab, although anti-PD-1 therapy uncommonly results in permanent toxicities.

The strategy of management of recurrences during and after adjuvant therapy is an evolving paradigm, with no clear evidence to guide treatment choices. Our opinion is to offer resection if the recurrent disease is resectable. The options for BRAF wild-type patients who relapse during treatment with anti-PD-1 agents include combination ipilimumab and nivolumab immunotherapy, single-agent ipilimumab or clinical trials. If the relapse occurs after completion of anti-PD-1 treatment, they could be offered a rechallenge of the same treatment or one of the above-mentioned options could be explored.

If the relapse occurs in BRAF mutant cohorts, depending on which agent they started with, their treatment can be switched to the other agent (i.e., targeted treatment to anti-PD-1 or vice versa).

\section{Conclusion}

The advent of targeted and immunotherapy treatments has ushered an era of effective adjuvant systemic therapy options for high-risk melanoma. Recent years have seen major developments in the adjuvant treatment of melanoma, with significant and clinically meaningful improvements in RFS consistently demonstrated across multiple large Phase III studies, using both immunotherapy agents and targeted treatments. Despite these promising early results, adjuvant treatment has to be used judiciously, incorporating careful consideration of appropriate patient selection, potential toxicities and the costs involved, and the key questions that are yet to be answered. 


\section{Future perspective}

Although the unprecedented magnitudes of the benefit seen with these new agents have quickly led to new standards of care for patients with stage 3 melanoma, new questions have arisen, such as whether combination anti-CTLA-4 and anti-PD-1 immunotherapy, now preferred in some metastatic contexts, might offer further improved outcomes when used in the adjuvant setting. The Checkmate 915 study is addressing this in an ongoing Phase III, randomized trial of adjuvant nivolumab combined with ipilimumab versus nivolumab monotherapy after complete resection of stage $3 \mathrm{~B} / \mathrm{C} / \mathrm{D}$ or stage 4 melanoma. This will assess the efficacy of combination immunotherapy therapy in the adjuvant setting.

Another question is whether the benefits of immunotherapy will translate to even earlier disease stages. The Keynote 716 study that has recently opened around the world and is randomizing resected stage $2 \mathrm{~B} / \mathrm{C}$ patients to 12 months of pembrolizumab or placebo will address this. These studies will provide further data to inform the role of immunotherapy in resected melanoma.

Neoadjuvant immunotherapy, in which systemic therapy is given before surgical resection, is also being studied, with recent results showing promise for this approach. The OPACIN-NEO study reported in 2018, examined neoadjuvant combination immunotherapy with three different regimens of ipilimumab and nivolumab [42]. A combination of ipilimumab at $1 \mathrm{mg} / \mathrm{kg}$ combined with nivolumab at $3 \mathrm{mg} / \mathrm{kg}$ given 3 weekly for two cycles was chosen to take forward into later phase studies, as this combination had a response rate of $77 \%$, with responders experiencing excellent outcomes to date.

\section{Executive summary}

\section{Background}

- The prognosis of stage 3 melanoma is considerably poor, with a 5-year overall survival (OS) of $43 \%$ in those with macroscopic lymph node (LN) metastases.

- The role of adjuvant therapy in patients with resected stage 3 melanoma is a rapidly evolving field.

- Recent advances in targeted therapies and immunotherapies have led to a number of practice changing adjuvant trials in resected stage 3 disease.

Interferon

- Interferon was shown to have only a modest survival benefit (3-4\% improvement in absolute 5 -year OS) as an adjuvant treatment for melanoma.

- This benefit, when balanced against toxicity and cost, has limited its uptake into routine practice.

Immunotherapy

- Ipilimumab when given up to 3 years, as adjuvant treatment was shown to have a $10 \%$ absolute improvement in OS and relapse-free survival (RFS), but toxicity and high treatment-related death rates have limited its widespread use.

- There is a significant improvement in RFS with nivolumab compared with ipilimumab, and pembrolizumab compared with placebo, with a better tolerable toxicity profile.

Targeted therapies

- The combination of oral dabrafenib and trametinib has shown RFS and OS improvements when compared with placebo in resected stage 3, BRAF mutant melanoma.

The American Joint Committee on Cancer staging system

- Due to changes in the American Joint Committee on Cancer staging system, adjuvant therapy has to be recommended with caution to patients with stage $3 \mathrm{~A}$ disease, due to the modest therapeutic benefits in the subgroup.

Completion LN dissection

- The adjuvant trials were designed in an era prior to changes in current practice with respect to completion LN dissection.

- There is a lack of evidence to guide adjuvant treatment in this group and requires ongoing investigation.

Future directions

- The role of adjuvant immunotherapy is being further explored in a few areas - neoadjuvant setting, earlier stages of disease and combination treatments.

\section{Author contributions}

The first author and final author have made significant contributions in the design and conception of the work and writing of the manuscript in addition to (a-d). All the coauthors listed above participated sufficiently in the work to take responsibility for the content. They have made substantial contributions to (a) the acquisition, analysis and interpretation of data for the work; (b) 
drafting the work or revising it critically for important intellectual content; (c) final approval of the version to be published and (d) agreement to be accountable for all aspects of the work in ensuring that questions related to the accuracy or integrity of any part of the work are appropriately investigated and resolved.

Financial \& competing interests disclosure

The authors have no relevant affiliations or financial involvement with any organization or entity with a financial interest in or financial conflict with the subject matter or materials discussed in the manuscript. This includes employment, consultancies, honoraria, stock ownership or options, expert testimony, grants or patents received or pending, or royalties.

No writing assistance was utilized in the production of this manuscript.

Open access

This work is licensed under the Attribution-NonCommercial-NoDerivatives 4.0 Unported License. To view a copy of this license, visit http://creativecommons.org/licenses/by-nc-nd/4.0/

\section{References}

Papers of special note have been highlighted as: $\bullet$ of interest

1. Balch CM, Gershenwald JE, Soong SJ et al. Multivariate analysis of prognostic factors among 2,313 patients with stage III melanoma: comparison of nodal micrometastases versus macrometastases. J. Clin. Oncol. 28(14), 2452-2459 (2009).

2. Castello G, Ruocco V, Satriano RA, Zarrilli D. Role of interferons in the therapy of melanoma. Melanoma Res. 1(5-6), 311-325 (1992).

3. Kirkwood JM, Strawderman MH, Ernstoff MS, Smith TJ, Borden EC, Blum RH. Interferon alfa-2b adjuvant therapy of high-risk resected cutaneous melanoma: the Eastern Cooperative Oncology Group Trial EST 1684. J. Clin. Oncol. 14(1), 7-17 (1996).

4. Kirkwood JM, Manola J, Ibrahim J, Sondak V, Ernstoff MS, Rao U. A pooled analysis of Eastern Cooperative Oncology Group and intergroup trials of adjuvant high-dose interferon for melanoma. Clin. Cancer Res. 10(5), 1670-1677 (2004).

5. Eggermont AM, Suciu S, Testori A et al. Long-term results of the randomized Phase III trial EORTC 18991 of adjuvant therapy with pegylated interferon alfa-2b versus observation in resected stage III melanoma. J. Clin. Oncol. 30(31), 3810-3818 (2012).

6. Eggermont AM, Suciu S, Testori A et al. Ulceration and stage are predictive of interferon efficacy in melanoma: results of the Phase III adjuvant trials EORTC 18952 and EORTC 18991. Eur. J. Cancer 48(2), 218-225 (2012).

7. McMasters KM, Edwards MJ, Ross MI et al. Ulceration as a predictive marker for response to adjuvant interferon therapy in melanoma. Ann. Surg. 252(3), 460-465 (2010).

8. Mocellin S, Lens MB, Pasquali S, Pilati P, Chiarion Sileni V. Interferon alpha for the adjuvant treatment of cutaneous melanoma. Cochrane Database Syst. Rev. (6), CD008955 (2013).

9. Melero I, Hervas-Stubbs S, Glennie M, Pardoll DM, Chen L. Immunostimulatory monoclonal antibodies for cancer therapy. Nat. Rev Cancer 7(2), 95-106 (2007).

10. Hodi FS, O'Day SJ, McDermott DF et al. Improved survival with ipilimumab in patients with metastatic melanoma. N. Engl. J. Med. 363(8), 711-723 (2010).

11. Robert C, Thomas L, Bondarenko L et al. Ipilimumab plus dacarbazine for previously untreated metastatic melanoma. N. Engl. J. Med. 364(26), 2517-2526 (2011).

12. O'Day SJ, Maio M, Chiarion-Sileni V et al. Efficacy and safety of ipilimumab monotherapy in patients with pretreated advanced melanoma: a multicenter, single-arm Phase II study. Ann. Oncol. 21(8), 1712-1717 (2010).

13. Eggermont AM, Chiarion-Sileni V, Grob JJ et al. Adjuvant ipilimumab versus placebo after complete resection of high-risk stage III melanoma (EORTC 18071): a randomised, double-blind, Phase 3 trial. Lancet Oncol. 16(5), 522-530 (2015).

14. Balch CM, Gershenwald JE, Soong SJ et al. Final version of 2009 AJCC melanoma staging and classification. J. Clin. Oncol. 27(36), 6199-6206 (2009).

15. Eggermont AM, Chiarion-sileni, Grob JJ et al. Prolonged survival in stage III melanoma with ipilimumab adjuvant therapy. N. Engl. J. Med. 375(1), 1845-1855 (2016).

16. McArthur GA, Ribas A. Targeting oncogenic drivers and the immune system in melanoma. J. Clin. Oncol. 31(4), $499-506$ (2013).

17. Hamid O, Robert C, Daud A et al. Safety and tumor responses with lambrolizumab (anti-PD-1) in melanoma. N. Engl. J. Med 369(2), 134-144 (2013).

18. Ribas A, Wolchok JD, Robert C et al. Updated clinical efficacy of the anti-PD-1 monoclonal antibody pembrolizumab in 411 patients with melanoma. Pigment Cell Melanoma Res. 27(1), 1223-1223 (2014).

19. Ribas A, Puzanov I, Drummer R et al. A randomized controlled comparison of pembrolizumab and chemotherapy in patients with ipilimumab-refractory melanoma. Presented at: the Society for Melanoma Research 2014 International Congress. Zurich, Switzerland, 13 November -16 November 2014. 
20. Robert C, Ribas A, Wolchok JD et al. Anti-programmed-death-receptor-1 treatment with pembrolizumab in ipilimumab-refractory advanced melanoma: a randomised dose-comparison cohort of a Phase 1 trial. Lancet 384(9948), 1109-1117 (2014).

21. Robert C, Long GV, Brady B et al. Nivolumab in previously untreated melanoma without BRAF mutation. N. Engl. J. Med 372(1), 320-330 (2015).

22. Weber J, Mandala M, Del Vecchio M et al. Adjuvant nivolumab versus ipilimumab in resected stage III or IV melanoma. N. Engl. J. Med. 377(19), 1824-1835 (2017).

- Key Phase III trial comparing immunotherapy treatments as adjuvant treatment in resected melanoma.

23. Eggermont AM, Blank CU, Mandala M et al. Adjuvant pembrolizumab versus placebo in resected stage III melanoma. N. Engl. J. Med. 378(19), 1789-1801 (2018).

- Pivotal Phase III trial examining the role of anti-PD1 treatment in the adjuvant setting.

24. Coens C, Bottomley A, Blank CU et al. Health-related quality of life for pembrolizumab versus placebo after complete resection of high-risk stage III melanoma: results from the EORTC 1325-MG/Keynote 054 trial - an international randomized double-blind Phase 3 trial. Presented at: ESMO Congress. Munich, Germany, 21 September 2018.

25. Grant A. McArthur and Antoni Ribas. Targeting oncogenic drivers and the immune system in melanoma. J. Clin. Oncol. 31(4), 499-506 (2013).

26. Chapman $\mathrm{PB}$, Hauschild $\mathrm{A}$, Robert $\mathrm{C}$ et al. Improved survival with vemurafenib in melanoma with BRAF V600E mutation. N. Engl. J. Med. 364(1), 2507-2516 (2011).

27. Hauschild A, Grob J, Demidov LV et al. Dabrafenib in BRAF-mutated metastatic melanoma: a multicentre, open-label, Phase 3 randomised controlled trial. Lancet 380(9839), 358-365 (2012).

28. Robert C, Karaszewska B, Schacter J et al. Improved overall survival in melanoma with combined dabrafenib and trametinib. N. Engl. J. Med. 372(1), 30-39 (2015).

29. Long GV, Stroyakovsky D, Gogas $\mathrm{H}$ et al. A randomized, double-blinded, Phase III study comparing the combination of dabrafenib and trametinib with dabrafenib and placebo as first-line therapy in unresectable or metastatic BRAFV600E/K mutant cutaneous melanoma. J. Clin. Oncol. 32(15), 9011-9011 (2015).

30. Dummer R, Ascierto PA, Gogas HJ et al. Encorafenib plus binimetinib versus vemurafenib or encorafenib in patients with BRAF-mutant melanoma (COLUMBUS): a multicentre, open-label, randomised Phase 3 trial. Lancet Oncol. 19(5), 603-615 (2018).

31. Larkin J, Ascierto PA, Dreno B et al. Combined vemurafenib and cobimetinib in BRAF-mutated melanoma. N. Engl. J. Med. 371(20), 1867-1876 (2014).

32. Long GV, Hauschild A, Santinami M et al. Adjuvant dabrafenib plus trametinib in stage III BRAF-mutated melanoma. N. Engl. J. Med. 377(19), 1813-1823 (2017).

- Pivotal Phase III trial showing the efficacy of targeted therapy in adjuvant melanoma treatment.

33. Schadendorf D, Hauschild A, Santinami M et al. Effect on health-related quality of life of adjuvant treatment with dabrafenib plus trametinib in patients with resected stage III BRAF-mutant melanoma. Presented at: ASCO Annual Meeting. IL, USA, 1 June-6 June 2018.

34. Hauschild A, Drummer R, Schadendorf D et al. Longer follow-up confirms relapse-free survival benefit with adjuvant dabrafenib plus trametinib in patients with resected BRAF V600-mutant stage III melanoma. J. Clin. Oncol. 36(35), 3441-3449 (2018).

35. Maio M, Lewis K, Demidov L et al. Adjuvant vemurafenib in resected, BRAFV600 mutation-positive melanoma (BRIM8): a randomised, double-blind, placebo-controlled, multicentre, Phase 3 trial. Lancet Oncol. 19(4), 510-520 (2018).

36. Amin MB, Edge S, Greene F et al. AJCC Cancer Staging Manual, 8th Edition. Springer, NY, USA (2017).

- Notable changes in the melanoma staging system.

37. Larkin J, Hauschild A, Santinami M et al. Dabrafenib plus trametinib as adjuvant treatment of resected BRAF-mutant stage III melanoma: findings from the COMBI-AD trial analyzed based on AJCC 8 classification. Presented at: ASCO Annual Meeting. IL, USA, 1 June-6 June 2018.

38. Eggermont AM, Blank CU, Mandala M et al. Prognostic and predictive value of AJCC 8, in the Phase 3 EORTC 1325/Keynote 054 trial of pembrolizumab vs placebo in resected stage 3 melanoma. Presented at: 15th International Congress of the Society for Melanoma Research. Manchester, UK, 24-27 October 2018.

39. Faries MB, Thompson JF, Cochran AJ et al. Completion dissection or observation for sentinel-node metastasis in melanoma. N. Engl. J. Med 376(1), 2211-2222 (2017).

- Practice changing approaches in management of sentinel node metastasis in melanoma.

40. Leiter U, Stadler R, Mauch C et al. Complete lymph node dissection versus no dissection in patients with sentinel lymph node biopsy positive melanoma (DeCOG-SLT): a multicentre, randomised, Phase 3 trial. Lancet Oncol. 17(6), 757-767 (2016).

41. Hamid O, Robert C, Daud A et al. Five-year survival outcomes for patients with advanced melanoma treated with pembrolizumab in KEYNOTE-001. Ann. Oncol. 30(4), 582-588 (2019).

42. Rozeman EA, Menzies AM, Van de Wiel BA et al. OpACIN-neo - a multicenter Phase 2 study to identify the optimal neo-adjuvant combination scheme of ipilimumab and nivolumab. Presented at: ESMO Congress. Munich, Germany, 21 September 2018.

- New evolving approaches in the management of locoregional disease. 